\title{
Eigenvalue problems for nonlinear conformable fractional differential equations with multi-point boundary conditions
}

\author{
Wengui Yang \\ School of Mathematics, Southeast University, Nanjing 210096 China \\ Ministry of Public Education, Sanmenxia Polytechnic, Sanmenxia 472000 China \\ e-mail: wgyang0617@yahoo.com
}

Received 29 Nov 2018

Accepted 13 Nov 2019

\begin{abstract}
In this paper, we investigate the eigenvalue problems for a class of nonlinear conformable fractional differential equations with multi-point boundary conditions by using the fixed point index theory and a variant of Krein-Rutman theorem. Finally, two examples are presented to show the effectiveness of our main result.
\end{abstract}

KEYWORDS: positive solution, multi-point boundary value problem, fixed point index theory

MSC2010: 34B18 26A33 34A34

\section{INTRODUCTION}

Since fractional differential equations can describe natural phenomena better than integer order differential equations, they have been extensively applied in various fields such as physics, chemistry, and engineering ${ }^{1-3}$. Fractional differential equations have attracted extensive attention, there exist a large number of published papers on fractional differential equations, we refer the readers to Refs. 4-6 and the references quoted therein. By using the properties of Green's functions and the KrasnoselskiiZabreiko fixed point theorem, Yang and Qin ${ }^{7}$ investigated the existence of positive solutions for a class of nonlinear Hadamard fractional differential equations with integral boundary conditions. Zhou ${ }^{8,9}$ established sufficient conditions for the existence of globally attractive solutions for the Cauchy problems of fractional differential (evolution) equations in abstract space and in the cases that the semigroup is compact as well as non-compact, respectively. By Avery-Peterson's fixed point theorem, Guo et $\mathrm{al}^{10}$ considered the existence of multiple positive solutions to a singular Caputo's fractional differential equations with infinite-point boundary conditions. Based on the direct calculation of quantum derivatives of the Lyapunov-Kravsovskii functionals and linear matrix inequality method, Yang et $\mathrm{al}^{11}$ obtained some sufficient conditions on delay-dependent and delay-independent asymptotical stability of Riemann-Liouville $q$-fractional neu- tral systems with mixed delays. Jiang ${ }^{12}$ obtained the eigenvalue interval for multi-point boundary value problems of fractional differential equations. By using some properties of the classical MittagLeffler functions, Nieto ${ }^{13}$ presented two new maximum principles for a linear fractional differential equation with initial or periodic boundary conditions. Based on Krasnosel'skii and Schauder fixed point theorems and monotone iterative technique, the authors ${ }^{14}$ studied existence and uniqueness of periodic solutions for a particular class of nonlinear fractional differential equations admitting its righthand side with certain singularities. By applying the Green's function and Guo-Krasnoselskii fixed point theorems, Yang ${ }^{15,16}$ investigated the positive solutions for the coupled integral and four-point coupled boundary value problem of nonlinear semipositone Hadamard fractional differential equations, respectively. Zhou et al ${ }^{17}$ considered the time-fractional reaction-diffusion equation with nonlocal boundary condition and established the existence and uniqueness of a weak solution of the proposed model using the Faedo-Galerkin method and compactness arguments.

In the past years, the concepts of fractional derivatives and integrals are given by the following considerable operators: the Riemann-Liouville fractional derivative and integral, Caputo fractional derivative and integral, Hadamard fractional derivative and integral as well as other fractional deriva- 
tive and integral. Recently, based on the basic limit definition of the derivative, Khalil et $\mathrm{al}^{18}$ gave a new well-behaved definition called the conformable fractional derivative. Later, Abdeljawad ${ }^{19}$ obtained chain rule, exponential functions, Gronwall type inequality, integration by parts, Taylor power series expansions as well as Laplace transforms of the conformable fractional derivative and integral. Conformable fractional differential equations are getting an increasing interest ${ }^{20-22}$. According to the Banach, Schaefer, and Rothe fixed point theorems and degree theory, Asawasamrit et $\mathrm{al}^{23}$ studied the existence and uniqueness of solutions for impulsive multi-orders Caputo-Hadamard fractional differential equations equipped with boundary and integral conditions. By using the Picone identity of conformable fractional differential equations on arbitrary time scales, Zhang and Sun ${ }^{24}$ obtained the Sturm-Picone comparison theorem of conformable fractional differential equations on time scales. Karayer et al ${ }^{25}$ introduced conformable fractional Nikiforov-Uvarov method by means of conformable fractional derivative and gave exact eigenstate solutions of Schrödinger equation for certain potentials in quantum mechanics. By using the equivalence transformation and the associated Riccati techniques, Tariboon and Ntouyas ${ }^{26}$ investigated oscillation for the solutions of impulsive conformable fractional differential equations. By using the comparison principle and the monotone iterative technique combined with the method of upper and lower solutions, Liu et $\mathrm{al}^{27}$ considered a coupled system of nonlinear conformable fractional differential equations. Yang et $\mathrm{al}^{28}$ obtained the exact solutions for nonlinear local fractional FitzHughNagumo and Newell-Whitehead equations by applying the traveling-wave transformation.

Motivated by the works mentioned above, we consider the following eigenvalue problem for nonlinear conformable fractional differential equation with multi-point boundary condition:

$$
\begin{aligned}
& x^{(\alpha)}(t)-\Theta x(t)=\lambda f(t, x(t)), \\
& x(a)=\sum_{j=1}^{n} \beta_{j} x\left(\xi_{j}\right), t \in[a, b], \alpha \in(0,1],
\end{aligned}
$$

where $x^{(\alpha)}$ is a conformable fractional derivative with $0<\alpha \leqslant 1, \Theta \geqslant 0, a<\xi_{1}<\xi_{2}<\cdots<\xi_{n} \leqslant b$, and the nonlinear term $f$ is a continuous function. The main purpose of this paper is to obtain the eigenvalue interval of a positive solution for the multi-point boundary value problem by using the fixed point index theory and a variant of KreinRutman theorem.

\section{PRELIMINARIES}

For the convenience of the readers, we present some necessary definitions and lemmas from conformable fractional calculus theory in this section.

Definition 1 [Refs. 18,19] The conformable fractional derivative starting from a point $a$ of a function $f:[a, \infty) \rightarrow \mathbb{R}$ of order $\alpha \in(0,1]$ is defined by

$$
T_{a}^{(\alpha)} f(t)=\lim _{\varepsilon \rightarrow 0} \frac{h\left(t+\varepsilon(t-a)^{1-\alpha}\right)-h(t)}{\varepsilon},
$$

for all $t>a, \alpha \in(0,1]$, provided that the limit exists. If $\lim _{t \rightarrow a^{+}} T_{a}^{(\alpha)} f(t)$ exists, then by definition, one has $T_{a}^{(\alpha)} f(a)=\lim _{t \rightarrow a^{+}} T_{a}^{(\alpha)} f(t)$.

Definition 2 [Refs. 18, 19] The conformable fractional integral starting from a point $a$ of a function $f:[a, \infty) \rightarrow \mathbb{R}$ of order $\alpha \in(0,1]$ is defined by

$$
I_{a}^{\alpha} f(t)=\int_{a}^{t}(s-a)^{\alpha} f(s) \mathrm{d} s .
$$

Next, we write $f^{(\alpha)}$ for $T_{a}^{(\alpha)} f(t)$ to denote $\alpha$-order conformable fractional derivative of $f(t)$.

Lemma 1 (Ref. 27) Let $\sigma \in C([a, b], \mathbb{R})$ and $\Theta \in \mathbb{R}$. The linear initial value problem

$$
\begin{aligned}
& x^{(\alpha)}(t)-\Theta x(t)=\sigma(t), \\
& x(a)=x_{0}^{*}, t \in[a, b], \alpha \in(0,1],
\end{aligned}
$$

has a unique solution

$$
\begin{aligned}
x(t)= & x_{0}^{*} \exp \left(\omega\left(t^{\alpha}-a^{\alpha}\right)\right) \\
& +\int_{a}^{t} s^{\alpha-1} \exp \left(\omega\left(t^{\alpha}-s^{\alpha}\right)\right) \sigma(s) \mathrm{d} s, \omega=\frac{\Theta}{\alpha} .
\end{aligned}
$$

Now we present a variant of Krein-Rutman theorem, which plays an important role in proving the main results.

Lemma 2 (Ref. 29) Let $K$ be a reproducing cone in a real Banach space $X, L: X \rightarrow X$ be a compact linear operator with $L(K) \subseteq K$, and $r(L)$ be the spectral radius of L. If $r(L)>0$, then there exists $\varphi \in K \backslash\{0\}$ such that $L \varphi=r(L) \varphi$.

Lemma 3 (Ref. 30) Let $X$ be a Banach space, $P$ be a cone in $X$ and $\Omega(P)$ be a bounded open subset in $P$. Suppose that $A: \overline{\Omega(P)} \rightarrow P$ is a completely continuous operator. Then the following results hold: 
(I) If there exists $u_{0} \in P \backslash\{0\}$ such that $u \neq A u+\lambda u_{0}$ for all $u \in \partial \Omega(P), \lambda \geqslant 0$, then the fixed point index $i(A, \Omega(P), P)=0$.

(II) If $0 \in \partial \Omega(P)$ and $A u \neq \lambda u$ for all $u \in \partial \Omega(P), \lambda \geqslant$ 1 , then the fixed point index $i(A, \Omega(P), P)=1$.

For convenience, we assume that the following conditions hold.

$\left(H_{1}\right) \Theta \geqslant 0, f \in C\left([a, b] \times \mathbb{R}, \mathbb{R}^{+}\right), a<\xi_{1}<\xi_{2}<$ $\cdots<\xi_{n} \leqslant b$.

$\left(H_{2}\right) \beta_{j}>0, j=1,2, \ldots, n, \sum_{j=1}^{n} \beta_{j} \exp \left(\omega\left(\xi_{j}^{\alpha}-\right.\right.$ $\left.\left.a^{\alpha}\right)\right)<1$, where $\omega=\Theta / \alpha$.

At the same time, we make the following definitions

$$
\begin{aligned}
f_{0} & =\liminf _{x \rightarrow 0^{+}} \inf _{t \in[a, b]} \frac{f(t, x)}{x}, \\
f^{\infty} & =\limsup _{x \rightarrow+\infty} \sup _{t \in[a, b]} \frac{f(t, x)}{x}, \\
\mu_{0} & =\frac{1}{r(L)} .
\end{aligned}
$$

\section{MAIN RESULTS}

Lemma 4 Let $\sigma \in C([a, b], \mathbb{R})$ and $\Theta \in \mathbb{R}$. The following multi-point boundary value problem

$$
\begin{aligned}
& x^{(\alpha)}(t)-\Theta x(t)=\sigma(t), \\
& x(a)=\sum_{j=1}^{n} \beta_{j} x\left(\xi_{j}\right), t \in[a, b], \alpha \in(0,1],
\end{aligned}
$$

has a unique solution

$$
\begin{aligned}
x(t)= & \frac{\exp \left(\omega\left(t^{\alpha}-a^{\alpha}\right)\right)}{1-\sum_{j=1}^{n} \beta_{j} \exp \left(\omega\left(\xi_{j}^{\alpha}-a^{\alpha}\right)\right)} \\
& \times \sum_{j=1}^{n} \beta_{j} \int_{a}^{\xi_{j}} s^{\alpha-1} \exp \left(\omega\left(\xi_{j}^{\alpha}-s^{\alpha}\right)\right) \sigma(s) \mathrm{d} s \\
& +\int_{a}^{t} s^{\alpha-1} \exp \left(\omega\left(t^{\alpha}-s^{\alpha}\right)\right) \sigma(s) \mathrm{d} s .
\end{aligned}
$$

Proof: Following from Lemma 1, we obtain the following integral equation

$$
\begin{array}{rl}
x(t)=\sum_{j=1}^{n} \beta_{j} x & x\left(\xi_{j}\right) \exp \left(\omega\left(t^{\alpha}-a^{\alpha}\right)\right) \\
& +\int_{a}^{t} s^{\alpha-1} \exp \left(\omega\left(t^{\alpha}-s^{\alpha}\right)\right) \sigma(s) \mathrm{d} s .
\end{array}
$$

Letting $t=\xi_{j}$ in (2), we get

$$
\begin{aligned}
& \sum_{i=1}^{n} \beta_{i} x\left(\xi_{i}\right)=\sum_{i=1}^{n} \beta_{i}\left(\sum_{j=1}^{n} \beta_{j} x\left(\xi_{j}\right) \exp \left(\omega\left(\xi_{i}^{\alpha}-a^{\alpha}\right)\right)\right. \\
&\left.+\int_{a}^{\xi_{i}} s^{\alpha-1} \exp \left(\omega\left(\xi_{i}^{\alpha}-s^{\alpha}\right)\right) \sigma(s) \mathrm{d} s\right) .
\end{aligned}
$$

Solving (3) with respect to $\sum_{j=1}^{n} \beta_{j} x\left(\xi_{j}\right)$, we have

$$
\begin{array}{r}
\sum_{j=1}^{n} \beta_{j} x\left(\xi_{j}\right)=\frac{1}{1-\sum_{j=1}^{n} \beta_{j} \exp \left(\omega\left(\xi_{j}^{\alpha}-a^{\alpha}\right)\right)} \\
\times \sum_{j=1}^{n} \beta_{j} \int_{a}^{\xi_{j}} s^{\alpha-1} \exp \left(\omega\left(\xi_{j}^{\alpha}-s^{\alpha}\right)\right) \sigma(s) \mathrm{d} s .
\end{array}
$$

Substituting (4) into (2), we obtain the desired result.

Let $X=C[a, b]$ with the norm $\|x\|=$ $\max _{t \in[a, b]}|x(t)|$ and $P=\{x \in X: x(t) \geqslant 0, t \in$ $[a, b]\}$. It is easy to see that $P \subset X$ is a reproducing cone. Define operators $A: X \rightarrow X$ and $L: X \rightarrow X$ as follows:

$$
\begin{aligned}
A x(t)= & \frac{\lambda \exp \left(\omega\left(t^{\alpha}-a^{\alpha}\right)\right)}{1-\sum_{j=1}^{n} \beta_{j} \exp \left(\omega\left(\xi_{j}^{\alpha}-a^{\alpha}\right)\right)} \sum_{j=1}^{n} \beta_{j} \\
& \times \int_{a}^{\xi_{j}} s^{\alpha-1} \exp \left(\omega\left(\xi_{j}^{\alpha}-s^{\alpha}\right)\right) f(s, x(s)) \mathrm{d} s \\
& +\lambda \int_{a}^{t} s^{\alpha-1} \exp \left(\omega\left(t^{\alpha}-s^{\alpha}\right)\right) f(s, x(s)) \mathrm{d} s,
\end{aligned}
$$

$$
\begin{aligned}
L x(t)= & \frac{\exp \left(\omega\left(t^{\alpha}-a^{\alpha}\right)\right)}{1-\sum_{j=1}^{n} \beta_{j} \exp \left(\omega\left(\xi_{j}^{\alpha}-a^{\alpha}\right)\right)} \\
& \times \sum_{j=1}^{n} \beta_{j} \int_{a}^{\xi_{j}} s^{\alpha-1} \exp \left(\omega\left(\xi_{j}^{\alpha}-s^{\alpha}\right)\right) x(s) \mathrm{d} s \\
& +\int_{a}^{t} s^{\alpha-1} \exp \left(\omega\left(t^{\alpha}-s^{\alpha}\right)\right) x(s) \mathrm{d} s .
\end{aligned}
$$

Obviously, $x \in P$ is a solution of boundary value problem (1) if and only if it is a fixed point of the operator $A . \quad x$ is said to be a positive solution of boundary value problem (1) if it satisfies boundary value problem (1) and $x \in P \backslash\{0\}$.

Lemma 5 Let $\lambda>0$. Then the operator $A: P \rightarrow P$ is completely continuous.

Proof: From the continuity of $f(t, x)$, we know that $A: P \rightarrow P$ is continuous. Let $G \subset P$ be bounded. There exist constants $\Delta, \Delta_{0}>0$ such that $\|x\| \leqslant \Delta_{0}$, $x \in G$ and $|f(t, x)| \leqslant \Delta,(t, x) \in[a, b] \times\left[0, \Delta_{0}\right]$. For 
$x \in G$, we obtain

$$
\begin{aligned}
|A x(t)| \leqslant & \lambda \Delta \exp \left(\omega\left(b^{\alpha}-a^{\alpha}\right)\right) \\
& \times\left(\frac{\exp \left(\omega\left(b^{\alpha}-a^{\alpha}\right)\right)}{1-\sum_{j=1}^{n} \beta_{j} \exp \left(\omega\left(\xi_{j}^{\alpha}-a^{\alpha}\right)\right)}\right. \\
& \left.\times \sum_{j=1}^{n} \beta_{j} \int_{a}^{\xi_{j}} s^{\alpha-1} \mathrm{~d} s+\int_{a}^{t} s^{\alpha-1} \mathrm{~d} s\right) \\
\leqslant & \frac{\lambda \Delta \exp \left(\omega\left(b^{\alpha}-a^{\alpha}\right)\right)}{\alpha} \\
& \times\left(\frac{\exp \left(\omega\left(b^{\alpha}-a^{\alpha}\right)\right) \sum_{j=1}^{n} \beta_{j}\left(\xi_{j}^{\alpha}-a^{\alpha}\right)}{1-\sum_{j=1}^{n} \beta_{j} \exp \left(\omega\left(\xi_{j}^{\alpha}-a^{\alpha}\right)\right)}\right. \\
& \left.+\left(b^{\alpha}-a^{\alpha}\right)\right)<\infty,
\end{aligned}
$$

which implies that $A(G) \subset P$ is bounded. For $x \in G$, $t_{1}, t_{2} \in[a, b]$ with $t_{1}<t_{2}$, we get

$$
\begin{aligned}
& \left|A x\left(t_{2}\right)-A x\left(t_{1}\right)\right| \\
& \leqslant \frac{\lambda \Delta \exp \left(\omega\left(b^{\alpha}-a^{\alpha}\right)\right) \sum_{j=1}^{n} \beta_{j}\left(\xi_{j}^{\alpha}-a^{\alpha}\right)}{\alpha\left(1-\sum_{j=1}^{n} \beta_{j} \exp \left(\omega\left(\xi_{j}^{\alpha}-a^{\alpha}\right)\right)\right)} \\
& \quad \times\left|\exp \left(\omega\left(t_{2}^{\alpha}-a^{\alpha}\right)\right)-\exp \left(\omega\left(t_{1}^{\alpha}-a^{\alpha}\right)\right)\right| \\
& \quad+\lambda \mid \int_{a}^{t_{2}} s^{\alpha-1} \exp \left(\omega\left(t_{2}^{\alpha}-s^{\alpha}\right)\right) f(s, x(s)) \mathrm{d} s \\
& \quad \quad-\int_{a}^{t_{1}} s^{\alpha-1} \exp \left(\omega\left(t_{1}^{\alpha}-s^{\alpha}\right)\right) f(s, x(s)) \mathrm{d} s \mid \\
& \leqslant \frac{\lambda \Delta \exp \left(\omega\left(b^{\alpha}-a^{\alpha}\right)\right) \sum_{j=1}^{n} \beta_{j}\left(\xi_{j}^{\alpha}-a^{\alpha}\right)}{\alpha\left(1-\sum_{j=1}^{n} \beta_{j} \exp \left(\omega\left(\xi_{j}^{\alpha}-a^{\alpha}\right)\right)\right)} \\
& \quad \times\left|\exp \left(\omega\left(t_{2}^{\alpha}-a^{\alpha}\right)\right)-\exp \left(\omega\left(t_{1}^{\alpha}-a^{\alpha}\right)\right)\right| \\
& \quad+\lambda \Delta \int_{a}^{t_{1}} s^{\alpha-1}\left|\exp \left(\omega\left(t_{2}^{\alpha}-s^{\alpha}\right)\right)-\exp \left(\omega\left(t_{1}^{\alpha}-s^{\alpha}\right)\right)\right| \mathrm{d} s \\
& \quad+\lambda \Delta \exp \left(\omega\left(b^{\alpha}-a^{\alpha}\right)\right) \int_{t_{1}}^{t_{2}} s^{\alpha-1} \mathrm{~d} s \\
& \quad \frac{\lambda \Delta \exp \left(\omega\left(b^{\alpha}-a^{\alpha}\right)\right) \sum_{j=1}^{n} \beta_{j}\left(\xi_{j}^{\alpha}-a^{\alpha}\right)}{\alpha\left(1-\sum_{j=1}^{n} \beta_{j} \exp \left(\omega\left(\xi_{j}^{\alpha}-a^{\alpha}\right)\right)\right)} \\
& \quad \times\left|\exp \left(\omega\left(t_{2}^{\alpha}-a^{\alpha}\right)\right)-\exp \left(\omega\left(t_{1}^{\alpha}-a^{\alpha}\right)\right)\right| \\
& \quad+\lambda \Delta \int_{a}^{t_{1}} s^{\alpha-1}\left|\exp \left(\omega\left(t_{2}^{\alpha}-s^{\alpha}\right)\right)-\exp \left(\omega\left(t_{1}^{\alpha}-s^{\alpha}\right)\right)\right| \mathrm{d} s \\
& +\frac{\lambda \Delta \exp \left(\omega\left(b^{\alpha}-a^{\alpha}\right)\right)}{\alpha}\left(t_{2}^{\alpha}-t_{1}^{\alpha}\right) . \\
& +
\end{aligned}
$$

From the fact that $\exp (\cdot)$, and $\exp \left(\omega\left(t^{\alpha}-a^{\alpha}\right)\right)$ and $\exp \left(\omega\left(t^{\alpha}-s^{\alpha}\right)\right)$ are uniformly continuous on $[a, b]$ and $[a, b] \times[a, t]$, respectively. Therefore, $A(G)$ is equicontinuous on $[a, b]$. By Arzela-Ascoli theorem, we get that $A$ is compact.
Lemma 6 The operator $L: P \rightarrow P$ and $r(L)>0$.

Proof: It is easy to see that $L: P \rightarrow P$. Taking $x \equiv 1$, we have

$$
\begin{aligned}
L x(t)= & \frac{\exp \left(\omega\left(t^{\alpha}-a^{\alpha}\right)\right)}{1-\sum_{j=1}^{n} \beta_{j} \exp \left(\omega\left(\xi_{j}^{\alpha}-a^{\alpha}\right)\right)} \\
& \times \sum_{j=1}^{n} \beta_{j} \int_{a}^{\xi_{j}} s^{\alpha-1} \exp \left(\omega\left(\xi_{j}^{\alpha}-s^{\alpha}\right)\right) \mathrm{d} s \\
& +\int_{a}^{t} s^{\alpha-1} \exp \left(\omega\left(t^{\alpha}-s^{\alpha}\right)\right) \mathrm{d} s \\
\geqslant & \frac{1}{1-\sum_{j=1}^{n} \beta_{j} \exp \left(\omega\left(\xi_{j}^{\alpha}-a^{\alpha}\right)\right)} \\
& \times \sum_{j=1}^{n} \beta_{j} \int_{a}^{\xi_{j}} s^{\alpha-1} \exp \left(\omega\left(\xi_{j}^{\alpha}-s^{\alpha}\right)\right) \mathrm{d} s \\
= & : \mathscr{L}^{>} 0, \\
L^{2} x(t)= & \frac{\exp \left(\omega\left(t^{\alpha}-a^{\alpha}\right)\right)}{1-\sum_{j=1}^{n} \beta_{j} \exp \left(\omega\left(\xi_{j}^{\alpha}-a^{\alpha}\right)\right)} \sum_{j=1}^{n} \beta_{j} \\
& \times \int_{a}^{\xi_{j}} s^{\alpha-1} \exp \left(\omega\left(\xi_{j}^{\alpha}-s^{\alpha}\right)\right) L x(s) \mathrm{d} s \\
& +\int_{a}^{t} s^{\alpha-1} \exp \left(\omega\left(t^{\alpha}-s^{\alpha}\right)\right) L x(s) \mathrm{d} s \\
\geqslant & \frac{1}{1-\sum_{j=1}^{n} \beta_{j} \exp \left(\omega\left(\xi_{j}^{\alpha}-a^{\alpha}\right)\right) \sum_{j=1}^{n} \beta_{j}} \\
& \times \int_{a}^{\xi_{j}} s^{\alpha-1} \exp \left(\omega\left(\xi_{j}^{\alpha}-s^{\alpha}\right)\right) L(s) \mathrm{d} s \\
\mathscr{L}^{2} . &
\end{aligned}
$$

Repeating the above progress, we observe $L^{n} x(t) \geqslant$ $\mathscr{L}^{n}$. So, $r(L)=\lim _{n \rightarrow \infty} \sqrt[n]{\left\|L^{n}\right\|} \geqslant \mathscr{L}>0$.

From Lemma 2, we can see that there exist $\varphi \in$ $P \backslash\{0\}$ such that

$$
L \varphi=r(L) \varphi .
$$

Lemma 7 Suppose that $0<f_{0} \leqslant \infty$. For $\mu_{0} / f_{0}<$ $\lambda<\infty$, there exists $r_{0}>0$ such that either $A$ has at least one fixed point in $\partial \Omega_{r}(P)$ or $i\left(A, \Omega_{r}(P), P\right)=0$, for all $0<r<r_{0}$, where $\Omega_{r}(P)=\{x \in P:\|x\|<r\}$.

Proof: From $\lambda>\mu_{0} / f_{0}$, there exists $r_{0}>0$ such that

$$
f(t, x) \geqslant \frac{\mu_{0}}{\lambda} x, \quad t \in[a, b], \quad x \in\left[0, r_{0}\right] .
$$

Assume that $A$ does not have a fixed point in $\partial \Omega_{r}(P)$, $0<r<r_{0}$. By using the condition (I) of Lemma 3, 
we only need to confirm that

$$
x \neq A x+k \varphi, \quad \forall x \in \partial \Omega_{r}(P), \quad k>0,
$$

where $\varphi$ is similarly defined as in (5). If not, there exists $\forall x_{0} \in \partial \Omega_{r}(P), k_{0}>0$ such that $x_{0}=A x_{0}+$ $k_{0} \varphi$. Combining with (6), we have $A x_{0} \geqslant \mu_{0} L x_{0}$ and $x_{0} \geqslant k_{0} \varphi$. From $L(P) \subset P$, we obtain $L x_{0} \geqslant$ $k_{0} r(L) \varphi=k_{0} \varphi / \mu_{0}$. So, we get $x_{0}=A x_{0}+k_{0} \varphi \geqslant$ $\mu_{0} L x_{0}+k_{0} \varphi \geqslant 2 k_{0} \varphi$. Repeating the above process, we can get that $x_{0} \geqslant n k_{0} \varphi$. This is a contradiction with $x_{0} \in \partial \Omega_{r}(P)$.

Lemma 8 Suppose that $0 \leqslant f^{\infty}<\infty$. For $0 \leqslant \lambda<$ $\mu_{0} / f^{\infty}$, there exists $\rho_{0}>0$ such that either $A$ has at least one fixed point in $\partial \Omega_{\rho}(P)$ or $i\left(A, \Omega_{\rho}(P), P\right)=1$, for all $\rho>\rho_{0}$, where $\Omega_{r}(P)=\{x \in P:\|x\|<\rho\}$.

Proof: From $\lambda<\mu_{0} / f^{\infty}$, we see that there exists $\rho_{1}>0$ such that

$$
f(t, x) \leqslant \frac{\mu_{0}-\varepsilon}{\lambda} x, \quad t \in[a, b], \quad x>\rho_{1},
$$

where $\varepsilon>0$ is small enough. According to the continuity of $f(t, x)$ on $[a, b] \times\left[0, \rho_{1}\right]$, there exists a constant $\Lambda_{0}>0$ such that $f(t, x) \leqslant \Lambda_{0} / \lambda,(t, x) \in$ $[a, b] \times\left[0, \rho_{1}\right]$. Therefore, we have

$$
f(t, x) \leqslant \frac{\mu_{0}-\varepsilon}{\lambda} x+\frac{\Lambda_{0}}{\lambda},(t, x) \in[a, b] \times \mathbb{R}^{+} .
$$

Let $\rho_{0}=\left\|\left(I /\left(\mu_{0}-\varepsilon\right)-L\right)^{-1} L\left(\Lambda_{0} /\left(\mu_{0}-\varepsilon\right)\right)\right\|$. Assume that $A$ does not have a fixed point in $\partial \Omega_{\rho}(P), \rho>$ $\rho_{0}$. Using the condition (II) of Lemma 3 , we need only to prove that $A x \neq k x, x \in \partial \Omega_{\rho}(P), k>1$. Otherwise, there exist $x_{0} \in \partial \Omega_{\rho}(P), k_{0}>1$ such that $A x_{0}=k_{0} x_{0}$. Combining with (7), we obtain $x_{0} \leqslant A x_{0} \leqslant\left(\mu_{0}-\varepsilon\right) L x_{0}+L \Lambda_{0}$. Furthermore, we have

$$
\left(\frac{1}{\mu_{0}-\varepsilon} I-L\right) x_{0} \leqslant L\left(\frac{\Lambda_{0}}{\mu_{0}-\varepsilon}\right) \text {. }
$$

According to the fact that $\left(I /\left(\mu_{0}-\varepsilon\right)-L\right)^{-1}=$ $\sum_{n=0}^{\infty}\left(\mu_{0}-\varepsilon\right)^{n+1} L^{n}$ and $L(P) \subseteq P$, we have

$$
\begin{aligned}
x_{0} & \leqslant\left(\frac{1}{\mu_{0}-\varepsilon} I-L\right)^{-1} L\left(\frac{\Lambda_{0}}{\mu_{0}-\varepsilon}\right) \\
& \leqslant\left\|\left(\frac{1}{\mu_{0}-\varepsilon} I-L\right)^{-1} L\left(\frac{\Lambda_{0}}{\mu_{0}-\varepsilon}\right)\right\|=\rho_{0} .
\end{aligned}
$$

This is a contradiction with $x_{0} \in \partial \Omega_{\rho}(P)$.

Next, we will give our main result in this paper.

Theorem 1 Let $0 \leqslant f^{\infty}<f_{0} \leqslant \infty$. Then the boundary value problem (1) has at least one positive solution for $\mu_{0} / f_{0}<\lambda<\mu_{0} / f^{\infty}$.
Proof: Let $0<r<\min \left\{r_{0}, \rho_{0}\right\}$ and $\rho>\max \left\{r_{0}, \rho_{0}\right\}$. Assume that $A$ does not have a fixed point in $\partial \Omega_{r}(P)$ and $\partial \Omega_{\rho}(P)$. By Lemma 7 and Lemma 8 , we get that $i\left(A, \Omega_{r}(P), P\right)=0$ and $i\left(A, \Omega_{\rho}(P), P\right)=1$. By using the fixed point index theory, we can obtained that $A$ has at least one fixed point in $\Omega_{\rho}(P) \backslash \overline{\Omega_{r}(P)}$.

\section{EXAMPLES}

Example 1 Consider the following boundary value problem

$$
\begin{aligned}
& x^{(\alpha)}(t)=\lambda \exp (t) \sqrt[3]{|x(t)|}, \\
& x(0)=\sum_{j=1}^{n} \beta_{j} x\left(\xi_{j}\right), t \in[0,1], \alpha \in(0,1] .
\end{aligned}
$$

Corresponding to the boundary value problem (1), we see that $M=0, f^{\infty}=0$ and $f_{0}=\infty$. From Theorem 1, we obtain that the boundary value problem (8) has at least one positive solution for $0<\lambda<\infty$, if $\beta_{j}>0, j=1,2, \ldots, n, \sum_{j=1}^{n} \beta_{j}<1$.

Example 2 Consider the following boundary value problem

$$
\begin{aligned}
& x^{(\alpha)}(t)=\lambda|x(t)|\left(3+\exp \left(\frac{1+t}{1+x^{2}(t)}\right)\right), \\
& x(0)=\sum_{j=1}^{n} \beta_{j} x\left(\xi_{j}\right), t \in[0,1], \alpha \in(0,1] .
\end{aligned}
$$

Corresponding to the boundary value problem (1), we see that $M=0, f^{\infty}=3$ and $f_{0}=3+$ e. From Theorem 1, we obtain that the boundary value problem (9) has at least one positive solution for $\frac{\mu_{0}}{3+\mathrm{e}}<\lambda<\frac{\mu_{0}}{3}$, if $\beta_{j}>0, j=1,2, \ldots, n, \sum_{j=1}^{n} \beta_{j}<1$.

Acknowledgements: This work was supported by the Scientific Research Foundation of Graduate School of Southeast University under Grant No. YBJJ1824 and the Key Scientific Research Program of Higher Education of Henan Province-Guidance Plan under Grant No. 16B110011. The authors would like to thank the anonymous referees for their constructive comments and suggestions that led to improvement of the original manuscript.

\section{REFERENCES}

1. Kilbas AA, Srivastava HM, Trujillo JJ (2006) Theory and Applications of Fractional Differential Equations, Elsevier, Amsterdam.

2. Sabatier J, Agrawal OP, Machado JAT (2007) Advances in Fractional Calculus: Theoretical Developments and Applications in Physics and Engineering, Springer-Verlag, Berlin. 
3. Zhou Y (2016) Fractional Evolution Equations and Inclusions: Analysis and Control, Elsevier, New York.

4. Zhou Y, Ahmad B, Alsaedi A (2017) Existence of nonoscillatory solutions for fractional neutral differential equations. Appl Math Lett 72, 70-74.

5. Yang W (2014) Positive solutions for nonlinear Caputo fractional differential equations with integral boundary conditions. J Appl Math Comput 44, 39-59.

6. Ahmad B, Ntouyas SK (2014) A fully Hadamard type integral boundary value problem of a coupled system of fractional differential equations. Fract Calc Appl Anal 17, 348-360.

7. Yang W, Qin Y (2017) Positive solutions for nonlinear Hadamard fractional differential equations with integral boundary conditions. ScienceAsia 43, 201-206.

8. Zhou Y (2018) Attractivity for fractional differential equations in Banach space. Appl Math Lett 75, 1-6.

9. Zhou Y (2018) Attractivity for fractional evolution equations with almost sectorial operators. Fract Calc Appl Anal 21, 786-800.

10. Guo L, Liu L, Wu Y (2016) Existence of positive solutions for singular fractional differential equations with infinite-point boundary conditions. Nonlinear Anal Model Control 21, 635-650.

11. Yang W, Alsaedi A, Hayat T, Fardoun HM (2019) Asymptotical stability analysis of Riemann-Liouville $q$-fractional neutral systems with mixed delays. Math Meth Appl Sci 42, 4876-4888.

12. Jiang W (2013) Eigenvalue interval for multi-point boundary value problems of fractional differential equations. Appl Math Comput 219, 4570-4575.

13. Losada J, Nieto JJ, Pourhadi E (2017) On the attractivity of solutions for a class of multi-term fractional functional differential equations. $J$ Comput Appl Math 312, 2-12.

14. Cabada A, Kisela T (2017) Existence of positive periodic solutions of some nonlinear fractional differential equations. Commun Nonlinear Sci Numer Simulat 50, 51-67.

15. Yang W (2015) Positive solutions for singular coupled integral boundary value problems of nonlinear Hadamard fractional differential equations. $J$ Nonlinear Sci Appl 8, 110-129.

16. Yang W (2015) Positive solutions for singular Hadamard fractional differential system with fourpoint coupled boundary conditions. J Appl Math Comput 49, 357-381.
17. Zhou Y, Shangerganesh L, Manimaran J, Debbouche A (2018) A class of time-fractional reaction-diffusion equation with nonlocal boundary condition. Math Meth Appl Sci 41, 2987-2999.

18. Khalil R, Horani MA, Yousef A, Sababheh M (2014) A new definition of fractional derivative. $J$ Comput Appl Math 264, 65-70.

19. Abdeljawad T (2015) On conformable fractional calculus. J Comput Appl Math 279, 57-66.

20. Batarfi H, Losada J, Nieto JJ, Shammakh W (2015) Three-point boundary value problems for conformable fractional differential equations. $J$ Funct Spaces 2015, ID 706383.

21. Benkhettou N, Hassani S, Torres DFM (2016) A conformable fractional calculus on arbitrary time scales. J King Saud Univ Sci 28, 93-98.

22. Chung WS (2015) Fractional newton mechanics with conformable fractional derivative. $J$ Comput Appl Math 290, 150-158.

23. Asawasamrit S, Ntouyas SK, Thiramanus P, Tariboon J (2016) Periodic boundary value problems for impulsive conformable fractional integro-differential equations. Bound Value Probl 2016, ID 122.

24. Zhang C, Sun S (2017) Sturm-Picone comparison theorem of a kind of conformable fractional differential equations on time scales. J Appl Math Comput 55, 191-203.

25. Karayer H, Demirhan D, Büyükkilic D (2016) Conformable fractional Nikiforov-Uvarov method. Commun Theor Phys 66, 12-18.

26. Tariboon J, Ntouyas SK (2016) Oscillation of impulsive conformable fractional differential equations. Open Math 14, 497-508.

27. Liu S, Wang H, Li X, Li H (2017) The extremal iteration solution to a coupled system of nonlinear conformable fractional differential equations. $J$ Nonlinear Sci Appl 10, 5082-5089.

28. Yang X-J, Feng G, Srivastava HM (2018) A new computational approach for solving nonlinear local fractional PDEs. J Comput Appl Math 339, 285-296.

29. Nussbaum RD (1981) Eigenvectors of nonlinear positive operators and the linear Krein-Rutman theorem. In: Fadell E, Fournier G (Eds), Fixed Point Theory, Lecture Notes in Math, 886, Springer, pp 309-330.

30. Guo D, Lakshmikantham V (1988) Nonlinear Problem in Abstract Cones, Academic Press, San Diego. 